\title{
Anxiety Controlling Wrist Band
}

\author{
Shruthi Sa ${ }^{a}$ Priya dharshini ${ }^{b}$, Ranjith Raj B ${ }^{c}$, Vaddi Lakshmi Satya Sai Sarojini ${ }^{d}$ \\ Ezhilarasi $\mathrm{P}^{\mathrm{e}}$, Rajesh Kannan $\mathrm{S}^{\mathrm{f}}$ \\ ${ }^{a} U G$ Scholar, Dept of ECE, St.Joseph's College of Engineering, Chennai, India \\ ${ }^{b} U G$ Scholar, Dept of ECE, St.Joseph's College of Engineering, Chennai, India \\ ${ }^{c} U G$ Scholar, Dept of ECE, St.Joseph's College of Engineering, Chennai, India \\ ${ }^{d} U G$ Scholar, Dept of ECE, St.Joseph's College of Engineering, Chennai, India \\ ${ }^{e}$ Associate professor, Dept of ECE, St.Joseph's College of Engineering, Chennai, India \\ ${ }^{f}$ Associate professor, Dept of ECE, St.Joseph's College of Engineering, Chennai, India
}

\begin{abstract}
Since the 1950s, suicide rate has been tripled among people aged between 15 and 24. Also, suicide is reported as the second most common cause of their deaths. Depression and anxiety are one of the major reasons for planting the thought of suicide in students' mind. People with anxiety disorders and stressful mind have excessive worry and fear about everyday situations. This intense worry and fear, though appears harmless, can reach its peak within minutes causing panic attacks. The main objective of our project is calming down the mind of people who are emotionally unstable and preventing the occurrence of any medical complications due to anxiety. Our innovation focuses on measuring the heart rate and controlling perturbation, anxiety, stress by applying acupressure at the H7 (Heart 7) point on the wrist crease.
\end{abstract}

Keywords. Anxiety; Stress; H7; Acupressure; healthcare; heartrate monitor; health tracking device; wrist band; COVID-19.

\section{Introduction}

Anxiety is a condition in which a person feels uneasy or nervous for an uncertain outcome. It is often caused by stress or trauma. Earlier it was considered to be a mental disorder but now it is common among many individuals. According to recent statistics over $40 \%$ of adult population is suffering from anxiety in USA. Acupressure is one of the ways to reduce anxiety. Anxiety controlling wrist band ${ }^{[1]}$ is one such device that measures our heart rate and automatically triggers pressure in pressure point on the wrist. This calms the people wearing it. It is also connected to a mobile application through Bluetooth. It gives you notification when your heart beat goes high. It also contains an oximeter that measures oxygen content in blood.

\section{Related Survey}

Heart rate is the primary parameter based on which the dysregulated emotions are monitored and tracked. Though there are a lot of devices used in monitoring these were 
emotions, controlling of these emotions with external devices is restricted. One of the effective and ancient methods of controlling these emotions manually is by stimulating certain part of the body for the respective function. In some of the existing models, a nausea control device which can be attached to the human wrist is described. Some devices use non-invasive nerve stimulation by passing electricity through two electrodes to stimulate the nerves. Another technique is to apply pressure to the acupuncture area either with the fingers or using a small rounded instrument (acupressure). Mobile devices for stimulation of certain acupuncture point are existing and are available for use. For example, the Sea Band (Sea-Band UK Ltd., USA) acupressure device is used for the treatment of nausea which stimulates the acupuncture point P6 and is specially designed for sea travellers. Other devices with similar application were also proposed, for example, Griffith, U.S. Pat. No. D274,557 [2], Giarratano, U.S. Pat. No. 5,078,728 [3], Humphrey, U.S. Pat. No. D356,433 ${ }^{[4]}$, Yoo (U.S. Pat. No. 5,774,424) ${ }^{[5]}$ describes an acupressure stimulator consisting of a pressure plate with nodules. In those methods, stimulation is done by giving pressure constantly. Scientific research have indicated that constant pressure for stimulation can be effective but it is not consistent and reduces over time, resulting in short-term benefits ultimately leading to the reappearance of the undesirable symptoms. Jacobs (U.S. Pat. No. 4,574,787) ${ }^{[6]}$ describes a device where a fluid vibrates and the vibration results in changing pressure. But it has the risk of liquid leaking and requires a separate vibration source. Fisher (U.S. Pat. No. 5,601,598) ${ }^{[7]}$ describes a method where the user has to periodically stretch the band to create changing pressure which is reported to be inconvenient by the users. Strumor (U.S. Pat. No. $5,607,749)^{[8]}$ describes a similar system to that of Jacob's where air is used instead of the fluid and the movement causes differential pressure. While these methods have some functionality, they need either bulky equipment or active user participation that limits the usage of the product. In some cases, patients need extended periods of treatment, and the current devices are inconvenient because they require constant operator action or immobility. Since it is required to treat these emotions during normal activities and instantly, without the user's attention, this band is prescribed.

\section{Proposed Methodology}

The Figure 1 gives the block diagram of the proposed anxiety reduction model.

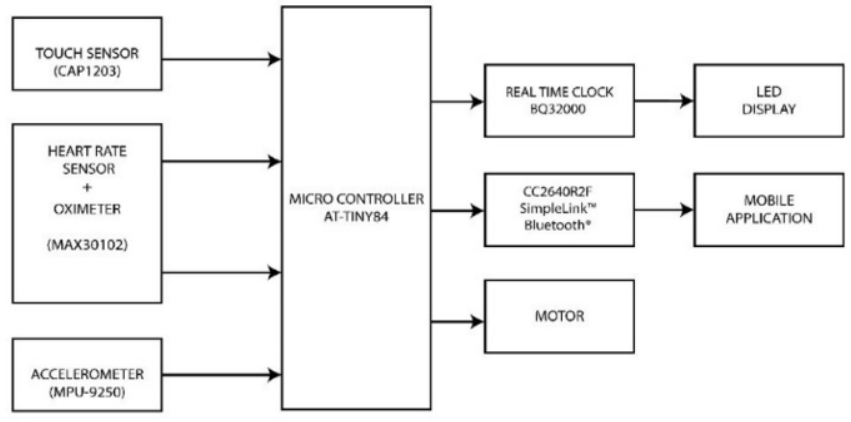

Figure 1 Block diagram of Proposed Anxiety Controlling Model 
The heart of the system is AT-TINY84 microcontroller which interfaces sensors ${ }^{[9]}$ and the outputs. The inputs to AT-TINY84 are given by the sensors, touch sensor, heart rate sensor, oximeter and accelerometer. MAX30102 is an integrated device that contains both pulse oximetry and heart-rate monitor. MPU-9250 is a 9-axis Motion Tracking device that combines a 3-axis gyroscope, 3-axis accelerometer, 3-axis magnetometer and a Digital Motion Processor all in a small package. Based on the inputs given to the controller, the clock, Bluetooth module and the motor of the acupressure system operates. The BQ32000 is the real time clock that displays time. CC2640R2F is the Bluetooth module which transmits the input data to the application. The motor drives the cam action of acupressure system.

\subsection{Sensors}

\subsubsection{Heart rate sensor and oximeter}

MAX30102 is an integrated device that contains both pulse oximetry and heart-rate monitoring sensor. It requires a low power supply of $1.8 \mathrm{~V}$. A standard I2C-compatible interface is used for communication. This methodology used by MAX30102 to measure the heart rate is called Photoplethysmography where light is incident in the skin and based on the reflection of the incident light, the measurements are made. One of the practical aspects of this approach is the possibility of differentiating the light reflected by the blood of an artery (produces an AC output) and other components of the body such as bones and tissues (produces a DC output). The sensor consists of a photo-diode that helps to convert the light to current which can be used as comprehensible data. In the device the MAX30102 sensor is installed as shown in Figure 2 thereby enabling the light source pass through the tissues to collect information about the heart rate and oxygen saturation. These values are then displayed to the user by connecting the device with a mobile application.

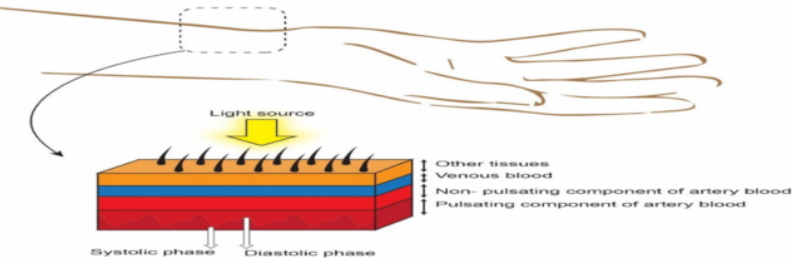

Figure 2. Light source pass through the tissues to collect information about the heart rate and oxygen saturation

\subsubsection{Touch sensor CAP1302}

Capacitive touch sensors are used in most of the portable devices like mobile phones and MP3 players. The dimension of CAP1302 is $2 \times 3 \mathrm{~mm}$. The CAP1203 includes Touch recognition which when detected, a status bit is set and an interrupt is generated. It comes with Active and Standby states, each with its own sensor input configuration controls. Power consumption in the Standby state varies depending on the enabled number of sensor inputs and number of outputs generated. The device communicates 
with a host controller using SMBus / I2C. Cap1302 features multiple pattern touch recognition that can sense touch simultaneously. If the pattern is observed, then the status bit is set and an interrupt is generated. The role of this sensor in the device is to make sure that the device is mounted firmly on the wrist. If the wristband is worn loosely, the acupressure setup will not be held firmly against the $\mathrm{H} 7$ point. Therefore, the switching $\mathrm{ON}$ of the whole acupressure system depends on the input from the CAP1302 sensor.

\subsubsection{Accelerometer, gyroscope, magnetometer}

MPU-9250 [10] is a multichip module which has a 3-Axis gyroscope, 3-Axis magnetometer and a 3-Axis accelerometer. The role of MPU-9250 in this device is the same as in the fitness bands. This sensor tracks the movement and orientation of the device thereby deriving the step count and the distance covered. It is also useful in tracking the rotation of the system. The LED display of the device displays the time when the device is found to be rotated. Therefore, the working of the LED display depends on the input from the MPU-9250. Apart from the above function, the major contribution of MPU-9250 in the device is to determine if the person is idle or not. The Acupressure system starts when the heart rate of the person is detected to be high. But, the increase in heart rate due to exercising, running and other physical activities should not trigger the acupressure system. The accelerometer serves this purpose of not switching ON the acupressure system unless the person is idle, even though the heart rate is rising beyond the normal limit.

\subsection{Acupressure}

The device of the present invention has a tiny DC motor 13 used to rotate the cam 16 across an acupressure nodule. The motor rotates with a speed $20 \mathrm{rpm}$ such that pressure is applied every 3 seconds. The motor starts rotating when the heart rate is found to be increased by the sensor and provides acupressure on the $\mathrm{H} 7$ acupuncture point 6 in order to treat anxiety. The cam will be in contact with the skin in the $\mathrm{H} 7$ acupuncture point 6 as shown in Fig 3. The microcontroller controls the motor $\mathbf{1 3}$ for this nodule. The cam will stimulate the $\mathrm{H} 7$ or Heart 7 acupuncture point when it is programmed to. It is programmed such a way that the motor stays idle normally and starts its operation only when the heart rate input to the microcontroller is abnormally high. The power supply for this motor is provided by the rechargeable battery. The conversion of rotational movement into linear movement, defined as the operation of cam is an application of the electromechanical principle. The whole acupressure setup is placed in the bottom layer of the device. A flexible sheet $\mathbf{1 0}$ seals the components against water, dust, etc. The compact dimension of the motor used $(11 \times 4 \times 4 \mathrm{~mm})$ and the dimension of cam being $1.5 \times 2 \times 1.5 \mathrm{~mm}$ helps the acupressure system to be occupied within the wearable device. The wrist point location and cam setup has been illustrated in Figure $3 \& 4$

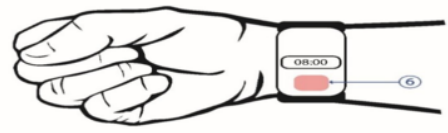

Figure 3. Location of the $\mathrm{H} 7$ or Heart 7 point on the wrist 


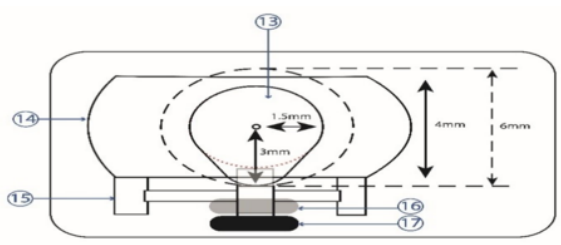

Figure 4. Acupressure system with cam setup

\subsection{Design}

The wrist band has a total length of $240 \mathrm{~mm}$ as illustrated in Figure 5. The main casing 1 is of the dimension of $10 \times 50 \times 30 \mathrm{~mm}$. The strap of the device is made using silicon while the curve-edged main case 1 is made up of hard plastic. The digital display $\mathbf{3}$ of the watch is at the center of the case, having the dimension of $10 \times 30 \mathrm{~mm}$ displaying the time in 24 hours clock-time.

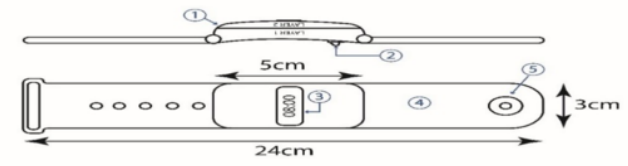

Figure 5. Vertical and Horizontal cross section of whole system

The lower layer of the device ${ }^{[11]}$ has the fabricated circuit containing a heart rate and pulse oximeter sensor $\mathbf{9}$, a touch sensor $\mathbf{1 1}$ occupying the left and center portion of the lower layer while the acupressure system $\mathbf{1 2}$ having a motor and cam occupies the rightmost of the base layer. The surface area of heart rate and pulse oximeter sensor is made substantial compared with other sensors so as to enhance the detection of pulse for various wrist sizes. The touch sensor $\mathbf{1 1}$ in the base layer is used to detect if the device is worn precisely. There are three different sizes available for the device: small, medium and large for different wrist sizes. Also, it comes with an instruction manual such that the watch must be worn in a specific region of wrist, making sure that the acupressure system $\mathbf{1 2}$ is focused on the $\mathrm{H7}$ point $\mathbf{6}$. The nodule $\mathbf{2}$ is not directly contacted with the skin and a flexible sheet $\mathbf{1 0}$ is used to cover the base of the nodule $\mathbf{2}$ in order to be sealed against water, dust, etc. This sheet $\mathbf{1 0}$ also serves the purpose of not causing any irritation or itchiness on the skin. The middle layer $\mathbf{8}$ of the device contains a rechargeable battery and a controller while the upper layer 7 of the main case has the accelerometer, LCD display 3 and a Bluetooth circuit. The controller controls the working of the motor 13 for acupressure system 12, the digital display 3 and the Bluetooth transmitter based on the inputs received by the touch, heart rate, pulse oximeter and the accelerometer. The function ${ }^{[12]}$ of the sensors and the controller is the same as that of a normal fitness band, which displays time, heartrate, step count, distance travelled and also which turns on when the gyroscope detects rotation or movement of the hand and connecting with an application in the mobile phone. The importance of accelerometer is that it helps in not raising an alarm message when the 
heart rate is increased during physical activities such as exercise, running, etc. The whole system has been illustrated in Fig 6. The enhancements made in this device includes the acupressure system 12 that provides with a reaction or solution to anxiety and nervousness and the pulse oximeter readings which helps to diagnose diseases like COVID-19.

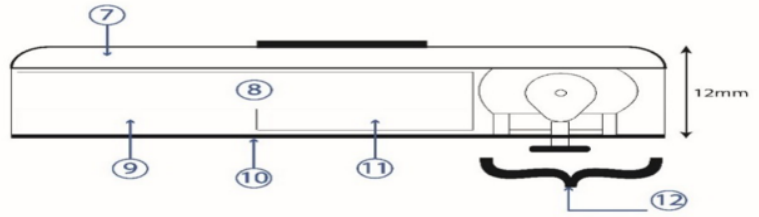

Figure 6. Vertical cross section of the system assembly

\subsection{Mobile Application}

For visualizing the sensor feeds, their values have to be updated to a User-Interface. The device uses Bluetooth MCU ${ }^{[13]}$ to send live sensor feeds to server which can be accessed through the mobile application. We have used Bluetooth low energy that is favourable considering the following features,

- Increased performance accuracy

- Compact system

- Covers Long Range (LE Coded PHYs) of 750m at High Speed (LE 2-Mbit PHY)

- Provides Longer battery life for the wireless applications

- Easy-to-use development environment

- Reduced cost

A mobile application is built using MIT application builder to visualize sensor measurements sent from our wearable device through Bluetooth. It allows communication between the device running the app and remote devices. The app is designed in such a way that it is easy to interact with people of all ages ${ }^{[14]}$. It also saves the records regularly in a remote database for offline processing. Anxiety can be caused due to many occasions i.e., shocks, stage fright etc. It causes abnormal heart rhythms that might go above 100 beats per minute which might cause serious health issues. So, it is necessary to monitor heart rates.

Our app continuously monitors the heart rate and gives alert notifications on abnormal heart rates. The world has been under threat due to the wide spread of Corona virus (COVID-19). Doctors have found a way to detect the virus using the oxygen saturation level in the blood of a person. The oximeter plays a crucial role in monitoring the oxygen levels and lets an individual know about the dropping levels. If the oxygen saturation content drops to $90 \%$ or below, those people are said to be affected by the Corona virus. Our device monitors the oxygen saturation levels and sends feeds to the app. An alert is sent to the users who are prone to COVID-19. In conclusion, typical features of the mobile application are,

- Oxygen saturation levels alert system

- Tracking water content and sleep 
- Heart rate and Oxygen level monitoring

- Available at different languages

\subsection{Results And Discussion}

Fifty students were enrolled in this study. Twenty-five students were given our prototype of anxiety controlling wrist band shown in Figure 7 (Group 1) whereas others were given normal wrist bands similar to Fitbit (Group 2). The students were given tasks to speak or perform on the dais facing other students. All the features were akin in both groups given in Table 1 except the weights in the Group 2 that were slightly more than the Group 1.

Table 1. Various Categories of students for experimentation

\begin{tabular}{lll}
\hline Categories & $\begin{array}{l}\text { Acupressure wrist band } \\
\text { Group } \\
\text { (Group 1) }\end{array}$ & $\begin{array}{l}\text { Normal wrist band Group } \\
\text { (Group 2) }\end{array}$ \\
\hline Age (Years) & $19 \pm 2$ & $19 \pm 2$ \\
Gender $(\mathrm{M} / \mathrm{F})$ & $10 / 15$ & $12 / 13$ \\
Height $(\mathrm{cm})$ & $151 \pm 5$ & $157 \pm 3$ \\
Weight $(\mathrm{Kg})$ & $60 \pm 5$ & $62 \pm 5$ \\
\hline
\end{tabular}

The students were made to wear our prototype on a daily basis which helped us to differentiate between health conditions of the two groups. After experimenting over two-month period, a significant change was seen in Group 1 than in Group 2. The former was able to express themselves in-front of large crowds without having any anxiety or stage fright. Also, they were able to communicate with strangers efficiently. Whereas the latter still had difficulty in expressing themselves and often developed nausea due to nervousness (anxiety) ${ }^{[15]}$. Though the Group 2 was able to track and monitor these emotions, they were not able to overcome it. It can be illustrated from Fig 8 that the number of students with acupressure wristband showed improved health conditions.

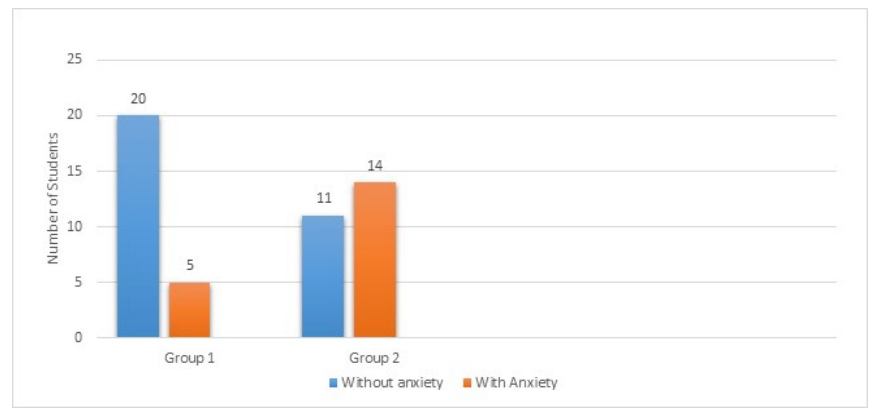

Figure 7 Anxiety level comparison chart of Normal fitbit and Proposed Prototype users

Acupressure is similar to acupuncture ${ }^{[16]}$ but without needles. It is based on the pathways of energy flow within the body called as meridians (traditional Chinese medicine). There are at least 14 meridian points connecting different organs. It is mostly used to manage symptoms of health complications (such as cancer, insomnia, headache, menstrual cramps and stress management). Some of the accupoints ${ }^{[17]}$ are, P6 point primarily used to treat nausea \& vomiting and $\mathrm{H} 7$ point is used for relaxing the body from various health issues such as panic attacks and stress. This point is located at the radial side of flexor carpi ulnaris (at the end of the palm). 


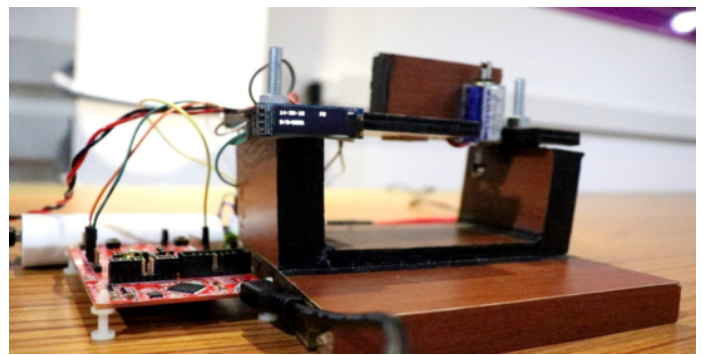

Figure 8. Automatic Prototype Acupressure Model

C1 Huatuo point is present at the top of the head and is an important juncture between brain and body. It is mostly used within Tam Healing system and Tong Ren Therapy. The user friendly application plays a major role for the device and various languages are available that can be modified based on users choice.

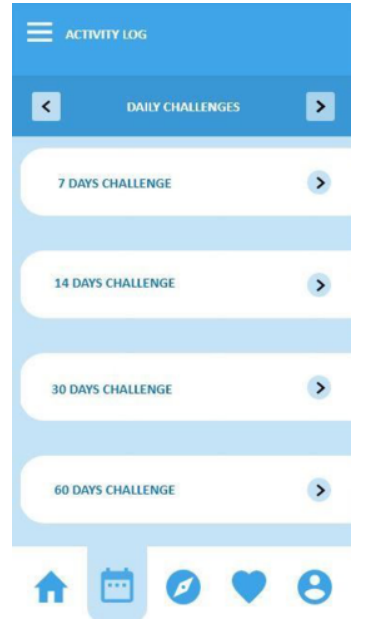

Figure 9. Activity log in App

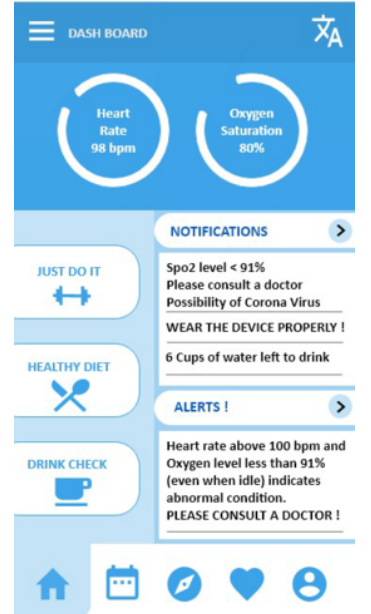

Figure 10. Application Dashboard

The dashboard or main page of our app is shown in Figure 10. Heart rate and SpO2 measurements are displayed in gauges with its measurements in bpm and percentages. If abnormal heart rate measurements are received, then alert message will be sent to the mobile phone through the app. Similarly, if SpO2 measurements are abnormal, alerts will be generated and will be directed to contact a doctor. Figure 9 is referred as the Activity log or workout log page. It consists of different workout challenge that differ from person to person depending upon their weight and height which must be provided as an initial requirement to the app.

For every hour from 9 A.M. to 9 P.M., we obtained heart rate measurements from a student while presenting a seminar and plotted the values in the form of a 2-D Area Chart with Time in hours along $\mathrm{X}$ axis and heart rate in beats per minute along $\mathrm{Y}$ axis shown in Figure 11. 


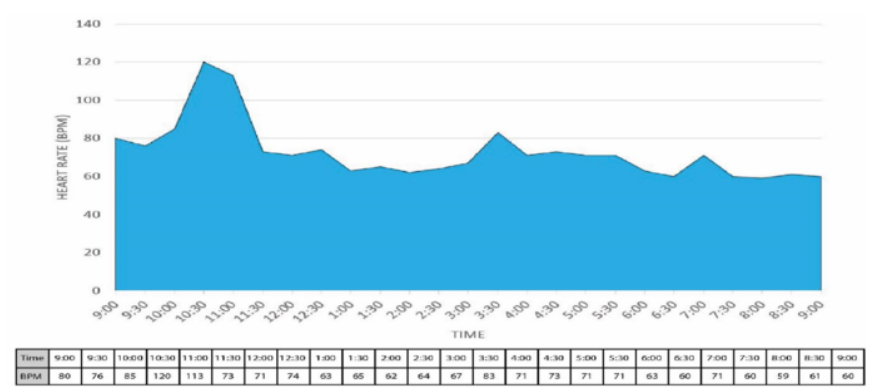

Figure 11. Heart rate measurements over a period of 12 hours

\section{Conclusion}

Many thought that the Asian method of acupressure as a suspicion earlier. However, we have demonstrated that it is a genuine method to prevent insomnia, anxiety, nausea and vomiting. The main objective of controlling anxiety is achieved by activating various acupressure points. Thus, anxiety-controlling wristband which makes use of the traditional method, is proved to be beneficial to assuage health and emotional illness through the course of experiments conducted on people suffering from anxiety.

\section{References}

[1] Mohamed Elgendi and Carlo Menon, "Assessing Anxiety Disorders Using Wearable Devices: Challenges and Future Directions", Journal of Brain Science, 9(3), pp.50, March 2019

[2] Richard Griffith, "Wristband for controlling nausea", United States Patent, 354,132, Des. 274,557, pp. 1-2, Jul. 3, 1984

[3] Thomas L. Grey, San Marcos, Robert J. Duffy, Poway, Robert C Giarratano, "Device for relief from morning sickness", United States Patent, 5,078,728, US 2004/0098037 A1, pp. 1-5, Jan. 7, 1992

[4] Doyce L Humphrey, "Elastic Wrist strap", United States Patent, 15,828, Des. 356,433, pp. 1-2, Mar. 21, 1995

[5] Tae Woo Yoo, “Acupressure stimulator for a watch", United States Patent, 784,571, Des. 5,774,424, pp.1- 4, Jun. 30, 1998

[6] H Barry Jacobs, “Acupressure apparatus”, United States Patent, 408,003, 4,574,787, pp. 1-5, Mar. 11, 1986

[7] Gary R Fisher, "Treatment device to aid in long-term cessation of smoking", United States Patent, 287,209, 5,601,598, pp. 1-17, Feb. 11, 1997

[8] Mathew A Strumor, "Ergonomic kinetic acupressure massaging system", United States Patent, 635,407, 5,607,749, pp. 1-13, Mar. 4, 1997

[9] Lee S.P., Haubrich N.R., Branche P.C., Li W, "Highly flexible, wearable, and disposable cardiac biosensors for remote and ambulatory monitoring", 31304288, PMC6550217, Jan 252018

[10] Inventions, "MPU-9250 Product Specification", PS-MPU-9250A-01, version 1.1, pp. 1-42, June. 20, 2016

[11] John G.Webster, "Medical Instrumentation Application and Design", 3rd Edition, Wiley India Edition, 2007

[12] L. E. Bertolucci, "Nausea Control Device”, United States Patent, 4,981,146, pp. 1-2, Jan. 1, 1991

[13] Texas Instruments, "32-bit Arm Cortex-M3 Bluetooth Low Energy wireless MCU with 128kB Flash and 275kB ROM (CC2640R2F)”, pp. 36-52, revised sept. 2020 
[14] Ting Zhang, Jiang Lu, Fei Hu and Qi Hao, "Bluetooth low energy for wearable sensor-based healthcare systems",14930130, 2014 IEEE Healthcare Innovation Conference (HIC), Seattle, WA, USA, 8-10 Oct. 2014

[15] Raghbir Singh Khandpur, "Handbook of Biomedical Instrumentation", $2^{\text {nd }}$ edition, TATA Mc GrawHill, New Delhi, 2003

[16] Joseph J.Carr and John M.Brown, "Introduction to Biomedical Equipment Technology", 4 ${ }^{\text {th }}$ edition, John Wiley and Sons, New York, 2004

[17] Basel Kikhia, Thanos G. Stavropoulos, Stelios Andreadis, Niklas Karvonen, "Utilizing a Wristband Sensor to Measure the Stress Level for People with Dementia”, Vol.16, Issue 12, May 2016 